\title{
Optical Cavity for Improved Performance of Solar Receivers in Solar-Thermal Systems
}

\author{
Lee Weinstein, Daniel Kraemer, Kenneth McEnaney, Gang Chen ${ }^{*}$ \\ Department of Mechanical Engineering, \\ Massachusetts Institute of Technology, Cambridge, MA 02139, USA
}

Abstract:

A principal loss mechanism for solar receivers in solar-thermal systems is radiation from the absorbing surface. This loss can be reduced by using the concept of directional selectivity in which radiation is suppressed at angles larger than the incident angle of the sunlight striking the absorber. Directional selectivity can achieve efficiencies similar to high solar concentration, without the drawbacks associated with large heat fluxes. A specularly reflective hemispherical cavity placed over the absorber can reflect emitted radiation back to the absorber, effectively suppressing emission losses. An aperture in the cavity will still allow sunlight to reach the absorber surface when used with point focus concentrating systems. In this paper the reduction in radiative losses through the use of a hemispherical cavity is predicted using ray tracing simulations, and the effects of cavity size and absorber alignment are investigated. Simulated results are validated with proof of concept experiments that show reductions in radiative losses of more than $75 \%$ from a near blackbody absorber surface. The demonstrated cavity system is shown to be capable of achieving receiver efficiencies comparable to idealized spectrally selective absorbers across a wide range of operating temperatures.

Highlights:

- A specularly reflective cavity placed over a solar absorber reduces radiative losses

- Cavity performance is sensitive to cavity size and cavity-absorber alignment

- Radiative loss reduction validated with proof of concept experiments

- Losses from near blackbody absorber reduced by over $75 \%$

- Receiver efficiency shown to be comparable to idealized wavelength selective absorber

Keywords: Solar receiver design, solar-thermal system, directional selectivity, radiative loss reduction

Nomenclature:

$A \quad$ area $\left(\mathrm{m}^{2}\right)$

C concentration ratio

(C) 2014. This manuscript version is made available under the Elsevier user license

http://www.elsevier.com/open-access/userlicense/1.0/ 


\section{Subscripts/superscripts:}

abs absorber

$a m b \quad$ ambient

cav cavity

$H \quad$ elevated (hot)

mis misalignment

$\lambda$ spectral

, directional

* $\quad$ effective with cavity 


\section{Introduction}

In addressing the challenge of a sustainable energy future, solar energy offers a promising solution due to its abundance (Smil, 2005). One method to harvest solar energy is using a solar-thermal system, in which solar energy is converted to thermal energy. This thermal energy can either be used directly (e.g., for water heating) or can be further converted to electrical, chemical or other forms of energy (Crabtree and Lewis, 2007). In solar thermal systems, sunlight which is incident on a solar collector is directed to a solar receiver and converted to thermal energy. Achieving high receiver efficiency, defined as the useful thermal energy delivered by the receiver divided by the solar energy incident on the receiver, is important to overall system performance. A solar receiver always consists of an absorber where solar energy is absorbed and converted to heat. Solar receivers may also include transmitting optics. For example, many receivers use transparent evacuated enclosures to eliminate convective losses. The efficiency $\eta_{\text {rec }}$ of such a solar receiver is given by

$$
\eta_{r e c}=\alpha \tau-\frac{\epsilon \sigma\left(T_{H}^{4}-T_{a m b}^{4}\right)}{C G}
$$

where $\alpha$ is absorptance of the absorber surface, $\tau$ is the transmittance through the receiver transmitting optics, $\epsilon$ is the emittance of the absorber surface, $\sigma$ is the Stefan-Boltzmann constant, $T_{H}$ is the elevated absorber temperature, $T_{a m b}$ is the ambient temperature, $C$ is the solar flux concentration ratio of the solar collector and $G$ is the solar power density input to the collector. The product $C G$ is the solar flux incident on the receiver. The first term of equation (1) is less than or equal to unity and represents losses due to incident sunlight failing to be absorbed (from being reflected away from the absorber, or from being absorbed at a location other than the absorber). The second term represents radiative losses from the absorber to the surroundings, normalized by incident solar power. This loss is primarily a function of absorber emittance, absorber temperature and the collector's optical flux concentration ratio. For receivers without the vacuum enclosure there would be an additional term representing convective losses from the absorber to the surroundings, however such receivers will not be considered in this paper.

Different strategies exist for designing high efficiency solar receivers, and the optimal strategy generally depends heavily on the intended operating temperature (Cao et al., 2014). For very low temperatures (e.g., $<100^{\circ} \mathrm{C}$ ), convective and radiative losses are small, so maximizing absorptance is the most important concern, and good efficiencies can be achieved using a simple black surface (Simon, 1976). For temperatures in the range of $100-300{ }^{\circ} \mathrm{C}$ convective losses become more important, but can be eliminated through the use of an evacuated transparent enclosure as mentioned earlier (Bermel et al., 2012; Mills, 2004). Using highly transparent enclosures and wavelength selective absorbers (surfaces with high absorptance in the solar spectrum but low emittance in the infrared (IR) spectrum) can lead to high efficiencies for these operating temperatures (Hiller et al., 2002; Selvakumar and Barshilia, 2012). For higher temperatures radiative losses dominate due to the fourth power dependence on absorber temperature and good efficiencies are typically achieved through the use of optical concentration, sometimes in combination with vacuum enclosures, wavelength selective surfaces, or other strategies (Fernández-García et al., 2010; Mills, 2004). The use of wavelength selective surfaces limits the receiver 
operating temperature due to diminishing reduction in emission losses from the overlap of the solar and IR spectra and reduces solar absorptance compared to blackbody receiver cavities. High optical concentrations significantly reduce the weighted radiative losses (second term of Eq. (1)) so having high solar absorptance becomes much more important to the receiver efficiency. Therefore, typically high optical concentration is used in combination with a black absorber or a blackbody cavity to efficiently operate at higher receiver temperatures (Prakash et al., 2009; Steinfeld and Schubnell, 1993). However, using high optical concentration ratios introduces complications such as large temperature gradients and complex heat exchangers required to handle a high heat flux.

Another way to improve the performance of solar systems is to use directional selectivity, which allows sunlight to be absorbed in specific directions but suppresses IR emission in other directions (Badescu, 2005; Blanco et al., 2004; Boriskina and Chen, 2014; Florescu et al., 2007). This can be achieved with photonic crystals (Hamam et al., 2011; Shen et al., 2014), grooved surfaces (Hollands, 1963; Perlmutter and Howell, 1963), and optical cavities (Braun et al., 2013; Gordon et al., 2004; Luque and Araújo, 1990). Using photonic crystals for solar thermal applications can be problematic as elemental diffusion between layers at high temperatures can ruin their radiative properties (Selvakumar and Barshilia, 2012). Adding grooves to a surface greatly increases the surface area, increasing radiative losses and making grooved surface absorbers non-ideal for solar receivers in practice. Optical cavities show promise, however there is an absence of reported experimental results for cavities with thermal applications.

In this work, we propose a solar receiver cavity design based on a specular mirror cavity that allows concentrated light to enter through an aperture and reduces the IR emission losses due to the aforementioned directional IR emission confinement. We first conceptually explore the receiver in terms of its directional selectivity to show its potential to improve efficiency in a manner similar to high concentration without having to deal with high heat fluxes and their associated challenges. The effectiveness of the cavity is predicted using ray tracing simulations, and these results are then validated by proof of concept experiments using an absorber suspended in a cavity. Results from the ray tracing simulations and physical experiments show good agreement, and demonstrate significant reductions in radiative losses from a near black surface. Predictions of possible efficiencies (Eq. (1)) for this receiver design are made and compared to other configurations.

\section{Cavity Receiver Concept}

The solar radiation striking the earth is close to collimated (the solar angular radius $\approx 0.0047 \mathrm{rad}$ ), thus a surface facing the sun absorbs direct sunlight at near normal incidence but emits IR radiation in all directions. If emission at large angles can be confined, then the emittance will be reduced without affecting the absorptance. Even under only one sun illumination, directional selectivity has the potential to raise receiver efficiency levels to those of high concentration systems (Badescu, 2005; Blanco et al., 2004).

Directional selectivity and optical concentration are similar strategies, because for both cases the goal is to increase the ratio of the solid angle over which sunlight is absorbed to the solid angle over which 
radiation is emitted. To understand how the two strategies are related, it is useful to look at the ratio $\epsilon / C$, which influences the relative importance of radiative losses to receiver efficiency, as seen in Eq. (1). For optimal performance this term should be minimized, which is why very efficient receivers use low emittance and high solar flux concentration. For an ideal directionally selective absorber with absorptivity of 1 for angles less than $\Psi$ and an absorptivity of 0 for angles greater than $\Psi$, the emittance is given by (Modest, 2013):

$$
\epsilon=2 \int_{0}^{\Psi} \sin \theta \cos \theta d \theta=\sin ^{2} \Psi
$$

For perfect concentrating optics delivering light within an acceptance angle $\Psi$, the maximum achievable concentration ratio, which is limited by conservation of etendue for the intercepted sunlight, is $\sin ^{2} \Psi / \sin ^{2} \theta_{\text {inc }}$ where $\theta_{\text {inc }}$ is the half angle of incident sunlight (Winston et al., 2005). Therefore the optimal ratio of $\epsilon / C$ is $\sin ^{2} \theta_{\text {inc }}$, and is independent of the acceptance angle of the system, showing that optical concentration and angular IR emission confinement are theoretically equivalent. In other words, a properly designed directionally selective system with the appropriate moderate solar flux concentration can achieve the same efficiency as a blackbody absorber under the maximum achievable solar concentration ratio. Operating at lower concentration ratios can be very beneficial as it avoids high heat fluxes which can cause large temperature gradients between the absorber surface and the intended delivery point of the heat (e.g., working fluid). A receiver using directional selectivity thus avoids inefficiencies from temperature gradients, complications from thermal stresses and the need for complicated heat exchanger designs, making directional selectivity a promising path to pursue.

Based on the above described concept this paper explores the directional selectivity of the proposed cavity receiver concept as shown in Fig. 1 . The idea of the cavity is simple: an absorber which emits in all directions but only receives sunlight over a small acceptance angle is covered by a reflective cavity with an aperture. The aperture allows sunlight over the acceptance angle to reach the absorber, while emitted radiation striking the cavity walls is specularly reflected back to the absorber to be reabsorbed. Since the radiation emitted at angles larger than the acceptance angle is confined, the absorber in the cavity effectively has directional selectivity. It is worth noting that many solar receiver cavities are "hot cavities," in that the entire cavity is at elevated temperature (Harris and Lenz, 1985), whereas in this cavity only the absorber is hot, a configuration which is compatible with solid state solar-conversion technologies such as solar thermoelectric generators and solar thermo-photovoltaics (Baranowski et al., 2012; Kraemer et al., 2011; Lenert et al., 2014). 
Figure 1 Operating principle of cavity. An aperture in the cavity allows sunlight to reach the absorber. Radiation emitted towards the cavity walls is specularly reflected back to the absorber to be reabsorbed, confining those losses.

The performance of the cavity is measured by the effective emittance $\epsilon^{*}$ of the absorber in the cavity. Due to the finite absorber size the directional emittance properties are not identical across the entire surface. Thus the directional emittance $\epsilon^{\prime}$ must be integrated over all solid angles and averaged over the entire absorber surface (this expression is simplified to only consider directional dependence in the polar angle $\theta$ ):

$$
\epsilon^{*}=\frac{2}{A_{a b s}} \int_{A_{a b s}} \int_{0}^{\pi / 2} \epsilon^{\prime}(\theta, x, y) \cos \theta \sin \theta d \theta d A
$$

where $A_{a b s}$ is the area of the absorber surface. If this system was used as a solar receiver, the radiative losses from the absorber would depend on its effective emittance, and thus the receiver efficiency can be calculated by:

$$
\eta_{r e c}=\alpha \tau-\frac{\epsilon^{*} \sigma\left(T_{H}^{4}-T_{a m b}^{4}\right)}{C G}
$$

with the only difference between this equation and Eq. (1) being the substitution of $\epsilon^{*}$. Therefore to improve receiver efficiency, the goal of the cavity is to reduce the effective emittance of the absorber. It has been shown that the ideal geometry for specularly reflecting radiation from a circular surface back to itself is a hemi-ellipsoid (Gordon et al., 2004). This paper investigates a hemispherical specularly reflective cavity, which can perform similarly to a hemi-ellipsoidal cavity (Weinstein, 2013), but is more readily available as a stock geometry. 


\section{Methods}

\subsection{Simulation}

It is intractable to determine the directional emissivity analytically at every point on the absorber, due to the complications created by the curved, specularly reflecting cavity. Thus, a ray tracing simulation code was developed in Matlab and was used to predict the cavity performance. Ray tracing is a Monte-Carlo technique in which many rays, representing bundles of radiative energy, are generated and their progression through a system is tracked. Ray tracing can be used to evaluate the overall radiative properties of a system (Howell, 1998). In this case, rays were generated originating at the absorber surface, and the effective emittance is determined by tallying the number of rays which returned to the absorber versus leaving through the aperture or being absorbed at the cavity walls.
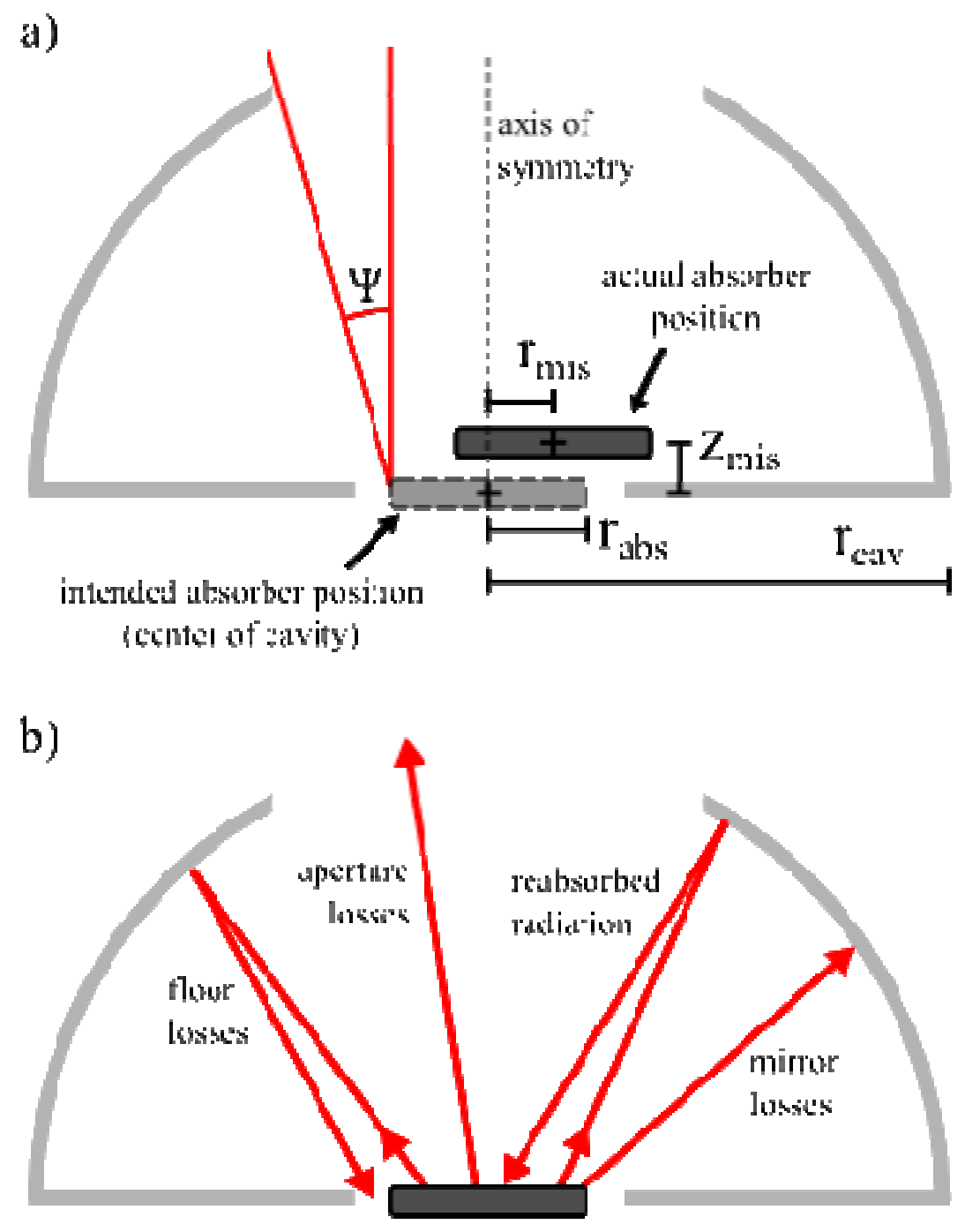

Figure 2 a) Simulated cavity geometry: cavity radius $r_{c a v}$, absorber radius $r_{a b s}$ and aperture acceptance angle $\Psi$ are all important. In practice radial misalignment $r_{m i s}$ and height misalignment $z_{m i s}$ can also influence performance. b) Different outcomes for rays emitted from the absorber: the desired outcome is that they are reabsorbed, but they can also be lost through the aperture and floor or absorbed by the mirrored cavity walls. 

parameters: the cavity size ratio, which is the ratio of the cavity radius to the absorber radius, and the acceptance angle of the cavity, which is important to determining aperture size and to determining the maximum solar concentration ratio usable with the cavity/absorber system. Additionally, the alignment of the absorber within the cavity can be very important to the performance, which can manifest as height misalignment $z_{m i s}$ or radial misalignment $r_{m i s}$. Various radiative properties (absorber emittance, specular and diffuse reflectances of all surfaces) can also be adjusted for different surfaces within the system. Figure $2 \mathrm{~b}$ shows the different outcomes for rays emitted from the absorber. Rays which are successfully confined are reflected back to the absorber where they are reabsorbed. Rays directed towards the aperture are lost to the environment ("aperture losses") and rays which reflect off the cavity but miss the absorber are lost through the floor of the cavity to the environment ("floor losses"). Ideally the cavity walls are perfectly reflecting but in practice they have finite absorptance and some rays are absorbed by the mirrored cavity walls ("mirror losses").

\subsection{Experiment}

Measuring total hemispherical emittance directly is very difficult as it requires isolating radiative losses from the surface being measured from all other heat transfer mechanisms, and this difficulty would only be compounded by attempting to use the cavity in conjunction with an existing measurement method (Palmer, 1995). Instead, the following comparative experimental procedure was developed to measure the effective emittance of the absorber within the cavity (see Fig. 3):

1. Attach the absorber surface to a heater and suspend the heater within a vacuum chamber such that there are minimal losses to the chamber. After evacuating the chamber, raise the heater to a specific elevated temperature and measure the power required to maintain that temperature.

2. Place the same heater/absorber structure in the vacuum chamber, now within the optical cavity. After evacuating the chamber, raise the heater to the same elevated temperature. Measure the power required to maintain the elevated temperature.

If there is good thermal contact between the absorber and heater, and if the whole heater/absorber structure is close to isothermal, then assuming a gray absorber with emittance $\epsilon$, the power inputs in the two cases $P_{1}$ and $P_{2}$ can be given by

$$
\begin{gathered}
P_{1}=Q_{l o s s}+A_{a b s} \sigma \epsilon\left(T_{H}^{4}-T_{a m b}^{4}\right) \\
P_{2}=Q_{l o s s}+A_{a b s} \sigma \epsilon^{*}\left(T_{H}^{4}-T_{a m b}^{4}\right)
\end{gathered}
$$

where $Q_{\text {loss }}$ is the backside losses from the heater/absorber structure, $A_{a b s}$ is the area of the absorber, $\sigma$ is the Stefan-Boltzmann constant, $\epsilon^{*}$ is the effective absorber emittance with the cavity, $T_{H}$ is the elevated absorber temperature and $T_{a m b}$ is the ambient temperature. If the experiment is designed such that the back side losses depend only on the elevated temperature and not on input power (e.g., power dissipation in the wires is negligible, etc.) then the back side losses should be the same for both cases. 
ai

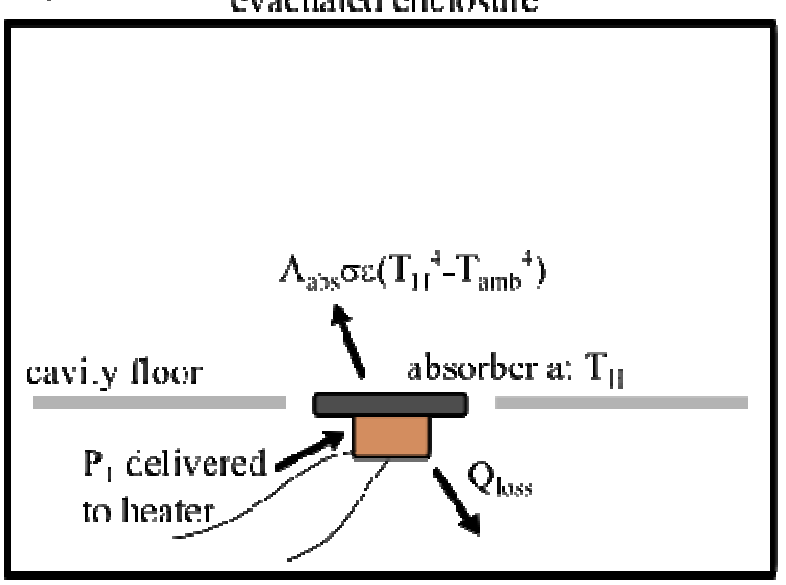

b)

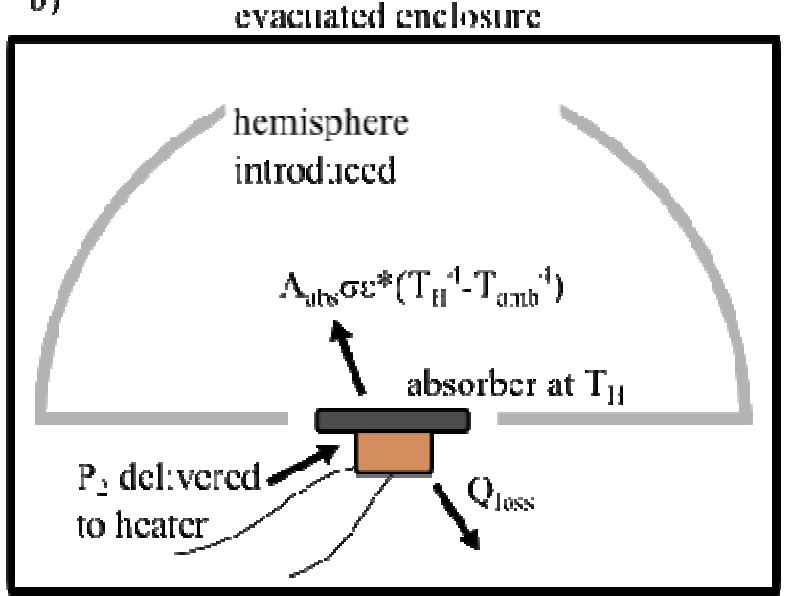

Figure 3 Experimental procedure for measuring the effective emittance of an absorber within an optical cavity: a) first the power required to maintain the absorber surface at $T_{H}$ is measured, then b) the power required to maintain the absorber surface at $T_{H}$ with the optical cavity is measured. The difference in power inputs gives the effective emittance as in Eq. (8)

Taking the difference in power inputs between the two cases thus calibrates out the back side losses and the reduction in effective emittance is given by

$$
\epsilon-\epsilon^{*}=\frac{P_{1}-P_{2}}{A_{a b s} \sigma\left(T_{H}^{4}-T_{a m b}^{4}\right)}
$$

If the emittance of the absorber is known, then the effective emittance of the absorber within the cavity is given simply by

$$
\epsilon^{*}=\epsilon-\frac{P_{1}-P_{2}}{A_{a b s} \sigma\left(T_{H}^{4}-T_{a m b}^{4}\right)}
$$

The set up shown in Fig. 4 was built in order to perform the experiment. The optical cavity being tested was a stock $9.2 \mathrm{~cm}$ inner diameter glass hemisphere from Edmund Optics, coated with an optically thick film of silver to form the reflective layer. The absorber surface was HE6 reference black paint on a $1 \mathrm{~cm}$ diameter copper block with a heater on the back side. The absorber temperature was measured with a 5-mil K-type thermocouple (SLE) embedded in the heater assembly. The electrical heater power was supplied and measured with a precision current source meter and digital multimeter by Keithley (Model: 2425/2010). The absorber/heater assembly was suspended on a ceramic pillar to minimize conductive losses. The pillar was mounted on a two axis linear stage to allow for precise absorber alignment. A machined aluminum plate was used as the cavity floor, which was suspended on threaded rods to allow for precise height adjustment between the cavity floor and absorber surface. Experiments were conducted in a Kurt J. Lesker box vacuum chamber with internal dimensions of $0.6 \mathrm{~m}$ per side at an operating pressure of approximately $10^{-4}$ torr. 

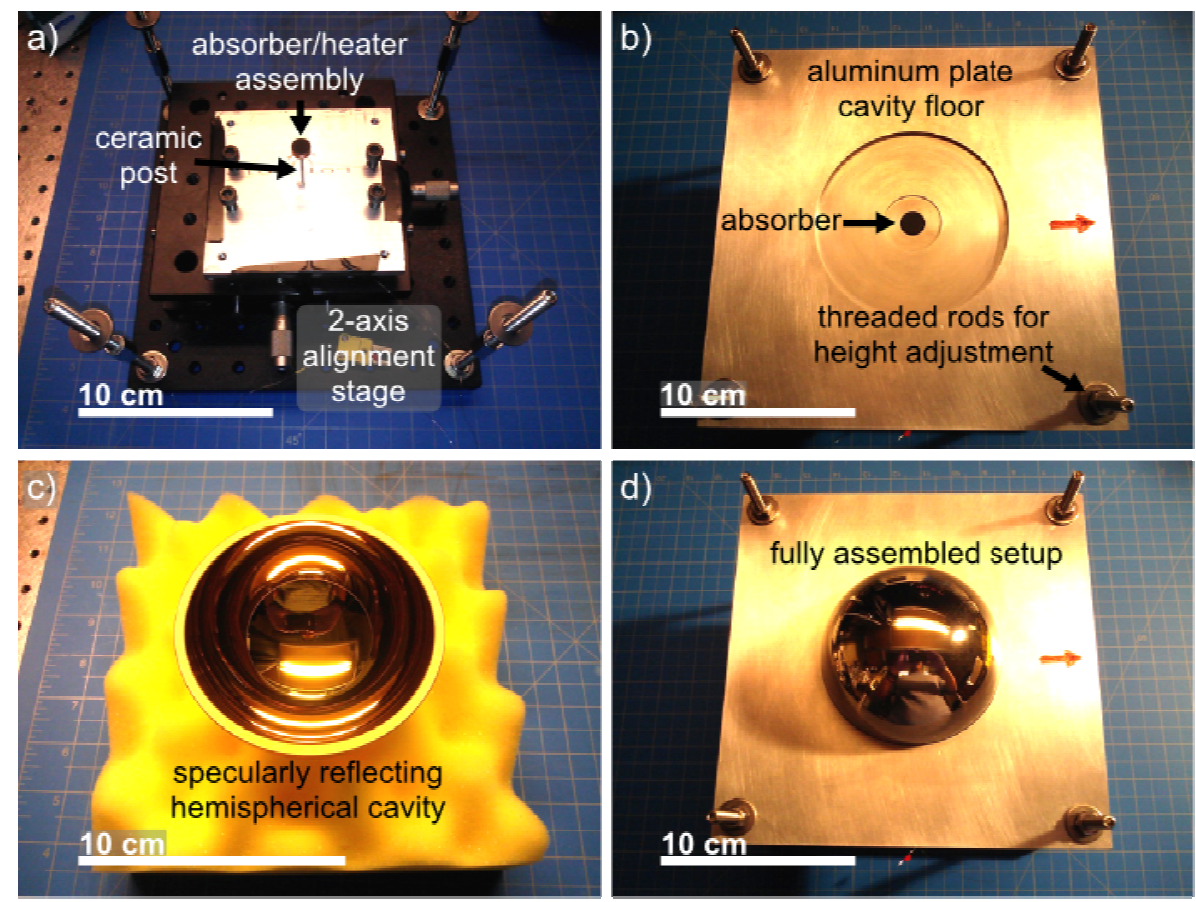

Figure 4 Photos of experimental setup a) heater and absorber surface suspended on ceramic pillar mounted to two axis linear stage b) absorber surface with cavity floor c) specularly reflective hemispherical dome d) fully assembled setup

Using a hemisphere with no aperture (as in Fig. 4) maximizes the experimental signal to noise ratio, and is therefore effective for investigating the effects of parameters other than aperture size. In order to test the effect of an aperture without fabricating a new cavity for each aperture size, "virtual apertures" were used. To simulate an aperture, an absorbing black plastic disk was suspended at the top of the hemisphere using magnets (see Fig. 5a). The radiation which is absorbed by the black disk is the same as the radiation which would be lost to an aperture of the same diameter as the disk, so in this manner the disk acts as a "virtual aperture". Switching between disks of different diameters allowed for rapid interchanging of different aperture sizes. Virtual apertures as used in the setup are shown in Fig. 5b. The plastic disks and magnets used as virtual apertures were measured in a Thermo Scientific FTIR Nicolet 6700 and showed specular reflectance of less than $5 \%$ for IR radiation with wavelengths in the range of 2-25 $\mu \mathrm{m}$. 


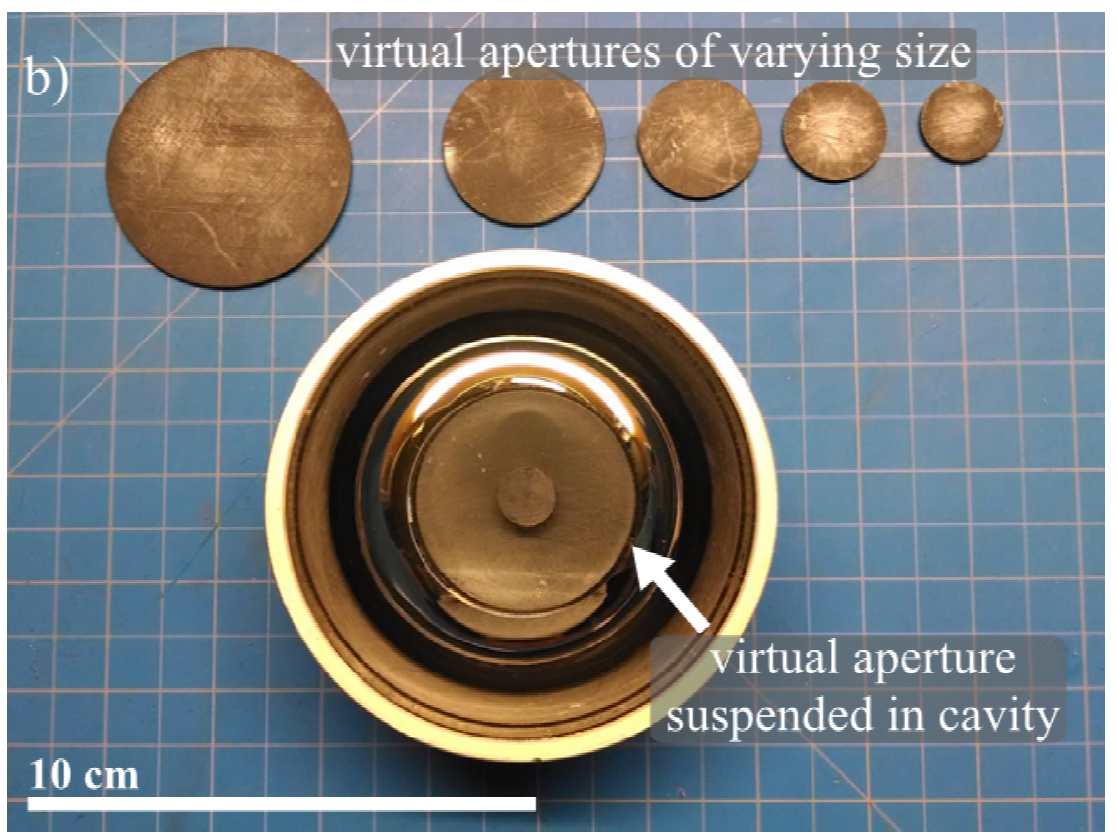

Figure 5 a) Diagram of virtual aperture concept: an absorbing black disk is suspended at the apex of the hemisphere and acts as a "virtual aperture." Radiation directed towards it will be absorbed and lost to the environment, as if there was an aperture of the same diameter as the disk. b) Photographs of the virtual apertures used for testing the effect of aperture size on cavity performance. Disks of varying diameter allow for rapid testing of different aperture sizes.

\section{$4 \quad$ Results and Discussion}

\subsection{Simulation}

Ray tracing simulations were performed to identify important parameters for cavity performance and inform design decisions. Simulations showed that effective emittance is very sensitive to the cavity size ratio, which is the ratio of cavity radius to absorber radius. Figure 6 shows effective emittance as a function of cavity size ratio for a blackbody absorber in a $95 \%$ specularly reflecting cavity with a $5^{\circ}$ acceptance angle. 
Figure 6 Effective emittance as a function of cavity size ratio predicted by ray tracing simulations for a blackbody absorber in a $95 \%$ specularly reflecting cavity with a $5^{\circ}$ acceptance angle. Total effective emittance is plotted (solid blue curve), as are contributions from aperture losses (dashed green curve), mirror losses (dash dotted red curve) and floor losses (dotted black curve).

As seen in Fig 6, use of the cavity can significantly reduce radiative losses. For a moderate cavity size ratio of 10 , the effective emittance drops to below 0.15 , representing a decrease in radiative losses of over $85 \%$ from the case of a black absorber with no cavity. For large cavity size ratios above 20 , effective emittance drops to below 0.1 . The cavity shows better performance for large cavity size ratios (corresponding to a cavity much larger than the absorber) for two reasons. First, for a given acceptance angle there is a smaller view factor to the aperture in larger cavities, resulting in fewer aperture losses. Second, in large cavities the hemispherical geometry more closely mimics the ideal hemi-ellipsoidal geometry, resulting in less reflected radiation missing the absorber and being lost through the cavity floor. Mirror losses are insensitive to cavity size at 0.05 in this case, matching the mirror absorptance. Thus, reductions in mirror absorptance lead to approximately equal reductions in effective emittance.

Another important geometric parameter of the cavity is the acceptance angle. Larger acceptance angles allow for higher concentration ratios to be used for the solar receiver system, but also result in more radiative losses from the absorber and thus higher effective emittance. In theory, for direct solar insolation on Earth, an acceptance angle of $5^{\circ}$ allows for concentration ratios in excess of $300 \mathrm{x}$, and an acceptance angle of $20^{\circ}$ allows for concentration ratios around 5000x (Winston et al., 2005). Figure 7 shows effective emittance as a function of cavity acceptance angle for a black absorber in a $95 \%$ specularly reflecting cavity with a cavity size ratio of 10 . 
Figure 7 Effective emittance as a function of acceptance angle predicted by ray tracing simulations for a blackbody absorber in a $\mathbf{9 5 \%}$ specularly reflecting cavity with a cavity size ratio of 10 . Total effective emittance is plotted (solid blue curve), as are contributions from aperture losses (dashed green curve), mirror losses (dash dotted red curve) and floor losses (dotted black curve).

As expected, an increase in acceptance angle increases effective emittance due to increased aperture losses, with minimal influence on mirror and floor losses. At a cavity size ratio of 10, effective emittance approaches 0.1 for very small acceptance angles near zero. Even for large angles, effective emittance is significantly reduced. An acceptance angle of $25^{\circ}$ yields an effective emittance of about 0.33 , corresponding to a reduction in radiative losses of two thirds from the case of a black absorber with no cavity.

The results above show significant reductions in effective emittance of an absorber within a reflective hemispherical cavity, however these results assume that the absorber is perfectly aligned at the center of the cavity. In practice, alignment of a small absorber within a large cavity is difficult, and can only be achieved to finite precision. Ray tracing simulations were also performed to predict the influence of misalignment on cavity performance. Figure 8 shows effective emittance as a function of absorber height misalignment normalized to absorber radius. 
Figure 8 Effective emittance as a function of normalized height misalignment predicted by ray tracing simulations for a blackbody absorber in a $95 \%$ specularly reflecting cavity with a $5^{\circ}$ acceptance angle. Total effective emittance is plotted for $r_{c a v} / r_{a b s}=5$ (solid blue curve), $r_{c a v} / r_{a b s}=10$ (dashed green curve) and $r_{c a v} / r_{a b s}=25$ (dotted black curve).

Figure 8 shows two important features of absorber height misalignment. First, it is clear that performance is very sensitive to height alignment. Changing the position of the absorber by 0.1 absorber radii can increase effective emittance by about 0.15 , more than doubling radiative losses in the large cavity cases. While not shown in the figure, the additional losses due to misalignment are floor losses, as changing the position of the absorber results in more of the reflected radiation missing the absorber. The second important feature to note is that the optimal absorber height is not at the equatorial plane of the hemisphere $\left(z_{m i s}=0\right)$, but slightly above. The ideal height is slightly higher than the center of the hemisphere because when the absorber is raised, the hemisphere more closely resembles the ideal oblate hemi-ellipsoidal geometry. The height which the absorber should be raised is a function of cavity size: larger cavities are closer to the ideal geometry, so the height to raise the absorber for ideal performance is smaller. It should be noted that for the results shown in all plots aside from Fig. 7 the absorber was placed at the ideal height, not at $z_{m i s}=0$.

Radial misalignment can also be important to the performance, and was also investigated using ray tracing simulations. Figure 9 shows effective emittance as a function of absorber radial misalignment normalized to absorber radius. 
Figure 9 Effective emittance as a function of normalized radial misalignment predicted by ray tracing simulations for a blackbody absorber in a $95 \%$ specularly reflecting cavity with a $5^{\circ}$ acceptance angle. Total effective emittance is plotted for $r_{c a v} / r_{a b s}=5$ (solid blue curve), $r_{c a v} / r_{a b s}=10$ (dashed green curve) and $r_{c a v} / r_{a b s}=25$ (dotted black curve).

The ideal radial alignment is always $r_{m i s}=0$, corresponding to the absorber being perfectly centered within the cavity. Performance is not quite as sensitive to radial alignment as height alignment, but radial alignment is still important. Deviations by 0.1 absorber radii increase effective emittance by about 0.1 , a significant increase for ideal effective emittances in the range of 0.05 to 0.2 . This increase in effective emittance comes from losses through the cavity floor when the misaligned absorber no longer intercepts radiation reflected from the cavity walls. While the effective emittance trend appears very similar for the different cavity size ratios, it is worth noting that the radial misalignment is normalized to the absorber radius, so achieving $r_{m i s} / r_{a b s}<0.05$ is more difficult for the higher cavity size ratio cases.

\subsection{Experiment}

In order to validate the ray tracing simulation results, proof of concept experiments as described in section 3.2 were also performed. The measured effective emittance of the $1 \mathrm{~cm}$ diameter HE6 reference black paint absorber in a $9.2 \mathrm{~cm}$ diameter reflective hemisphere is shown in Fig. 10 for various elevated temperatures and compared to ray tracing simulations for the same conditions. 


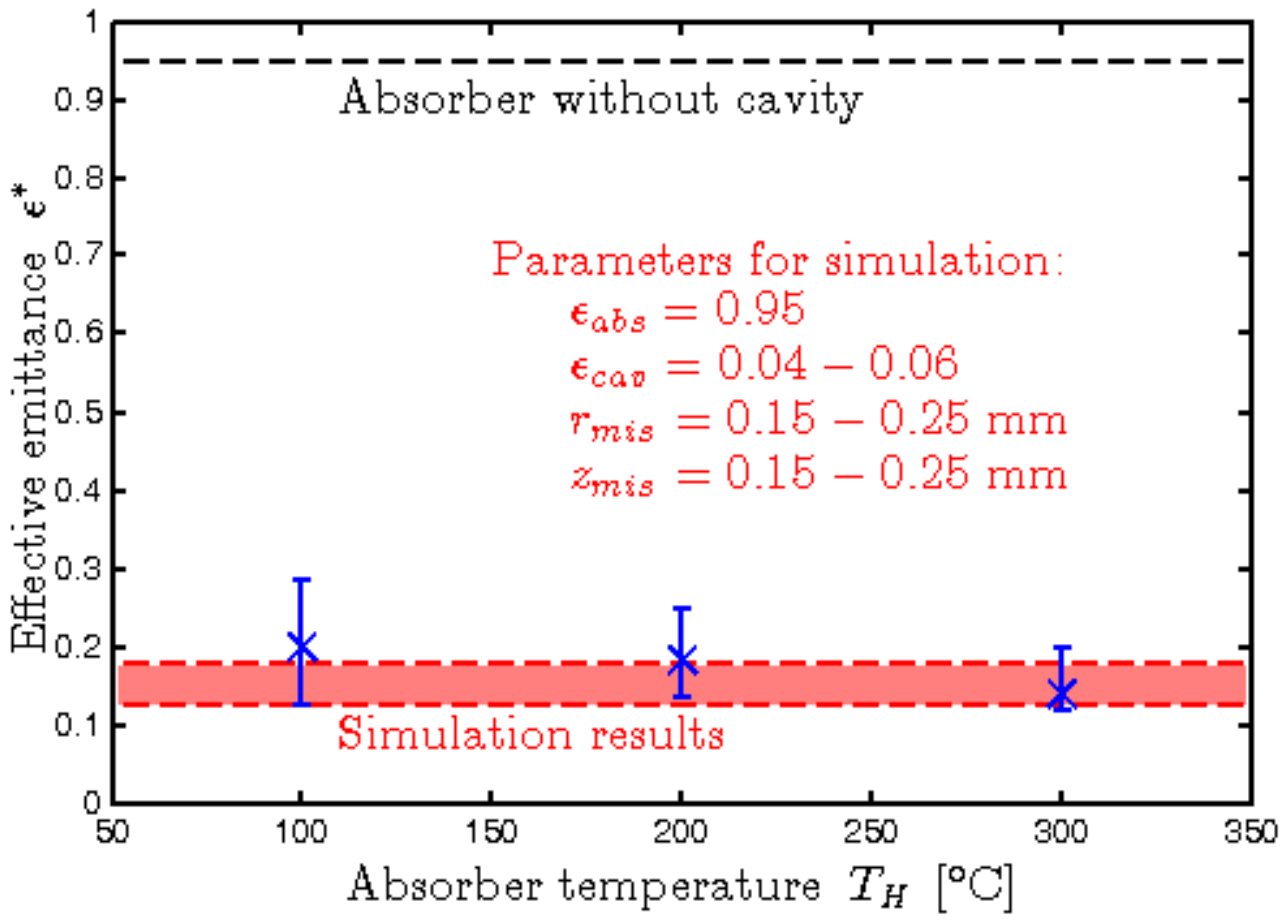

Figure 10 Measured effective emittance of near black absorber with cavity using prototype system as a function of absorber temperature. Error bars include systematic error and 3 standard deviation statistical error. Ray tracing simulation results are shown (red shaded region) for the same cavity geometry for comparison.

Measured effective emittance was significantly reduced with the use of the cavity, from the reference value of 0.95 (Brandt et al., 2008) to below 0.2 , an almost $80 \%$ reduction in radiative losses. These results show reasonable agreement with the effective emittance of $0.125-0.18$ predicted by ray tracing simulations for the same cavity geometry and a range of realistic cavity reflectances and absorber alignments. The expected dependence on absorber temperature is weak because none of the relevant radiative properties (emittances and reflectances) should change radically over the range of absorber temperatures investigated. Uncertainty at low temperatures is dominated by statistical error associated with taking the difference between two relatively similar numbers (power input with and without the cavity) while uncertainty at high temperature is dominated by systematic error associated with the large differences in power input for the cavity and no cavity cases. The remainder of the reported experimental results use data for an absorber temperature of $300^{\circ} \mathrm{C}$ as this temperature has the smallest overall uncertainty.

Most of the physical experiments were performed using a reflective hemispherical cavity with no aperture in order to maximize signal to noise ratio. As the role of aperture size on performance is important, the effect of aperture size was still investigated using the virtual aperture system discussed in section 3.2. Figure 11 shows measured effective emittance as a function of aperture acceptance angle for the virtual apertures. As expected, effective emittance increases with larger acceptance angles, as more radiation is lost through the larger apertures. Measured effective emittances show very good 
agreement with those predicted by ray tracing simulations for the range of acceptance angles investigated.

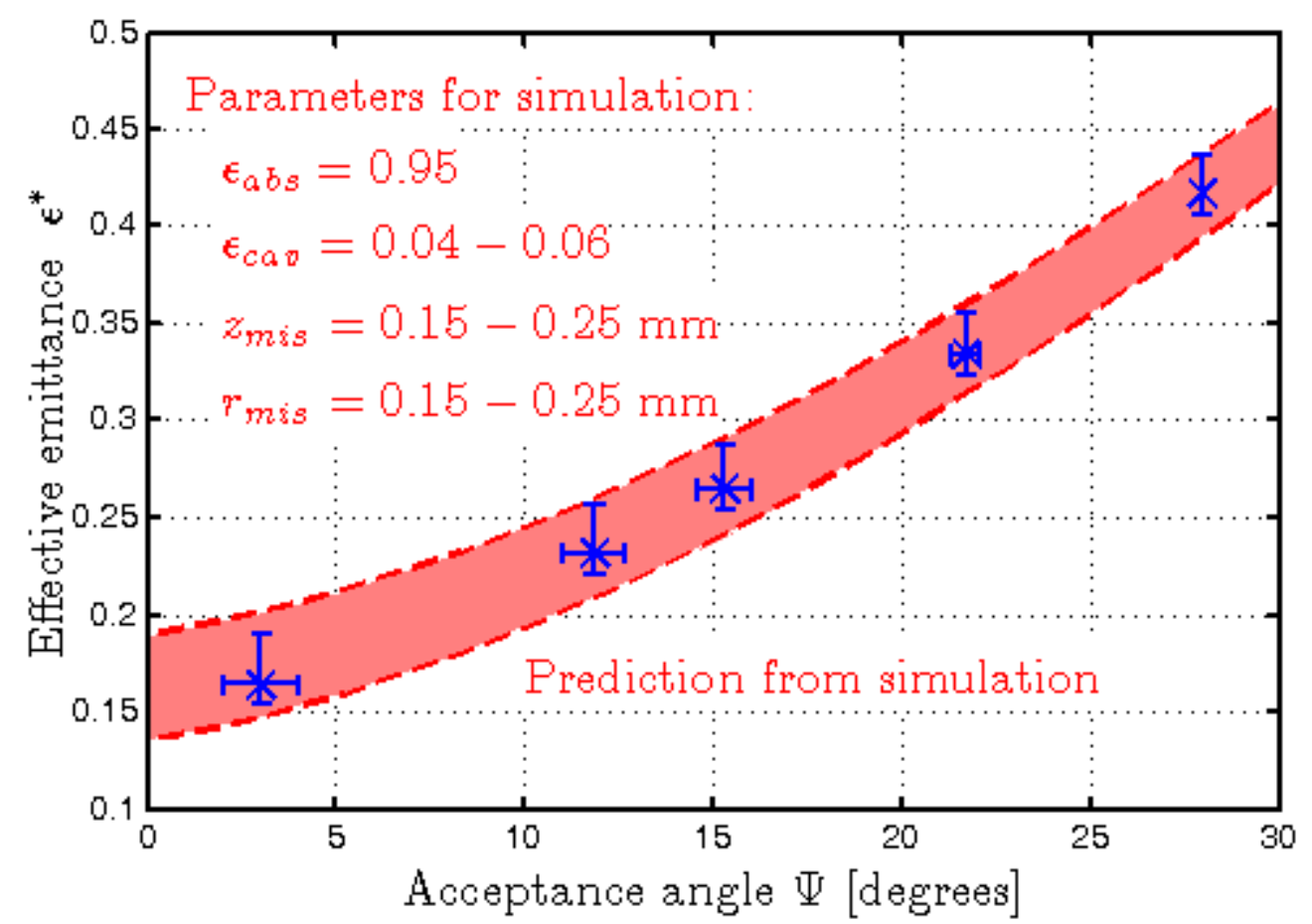

Figure 11 Measured effective emittance of near black absorber with cavity using prototype system as a function of aperture acceptance angle. Y-axis error bars include systematic error and 3 standard deviation statistical error, $\mathbf{x}$-axis error bars account for smaller virtual apertures not being flush with the top of the cavity. Ray tracing simulation results are shown (red shaded region) for the same cavity geometry for comparison.

As simulations showed that absorber alignment was very important to cavity performance, the experimental setup was designed to allow for precise positioning of the absorber relative to the reflective hemisphere. Measured effective emittance as a function of absorber height position in the cavity is shown in Fig. 12. Measured results show good agreement with predictions from ray tracing simulations, and the high sensitivity to height alignment is confirmed, as measurable differences in performance are observed for changes in alignment as small as $0.2 \mathrm{~mm}$ in the proof of concept experiment. 
Figure 12 Measured effective emittance of near black absorber with cavity using prototype system as a function of absorber height misalignment. Y-axis error bars include systematic error and 3 standard deviation statistical error, X-axis error bars denote precision which can reasonably be achieved for this system. Ray tracing simulation results are shown (red shaded region) for the same cavity geometry for comparison.

Effective emittance was also measured as a function of radial alignment and the results are shown in Fig. 13. Measured results again show reasonable agreement with predictions from ray tracing simulations. Performance is sensitive to radial alignment, however not as sensitive as height misalignment. Measurable differences in performance occur for changes in alignment as small as $0.5 \mathrm{~mm}$ in the proof of concept experiment. It should be noted that the mirrored hemisphere surface was lightly scratched before performing the radial alignment experiments, which could explain why the measured effective emittance values are slightly higher than the predictions from simulation. 
Figure 13 Measured effective emittance of near black absorber with cavity using prototype system as a function of absorber radial misalignment. Y-axis error bars include systematic error and 3 standard deviation statistical error, X-axis error bars denote precision which can reasonably be achieved for this system. Ray tracing simulation results are shown (red shaded region) for the same cavity geometry for comparison.

\subsection{Effect on receiver efficiency}

In order to appreciate how a reduction in emittance leads to increased receiver efficiency, the receiver efficiency is plotted as a function of absorber temperature for various receiver systems in Fig. 14. These efficiencies were calculated using Eq. (4), assuming the receiver is exposed to ASTM AM1.5 Direct + Circumsolar insolation (ASTM G, 2003) with a solar flux concentration ratio of 100. An effective emittance of 0.2 was used for the blackbody absorber with an optical cavity, which was demonstrated to be achievable for cavity receivers compatible with the concentration ratio of 100 (see Fig. 11). For the spectrally selective absorbers, the absorptance is treated as a step-function from 1 to 0 at an optimized transition wavelength (Cao et al., 2014) and a step function from 0.95 to 0.05 at a wavelength of $1.8 \mu \mathrm{m}$ for an ideal and more realistic surface, respectively. Transmittance is assumed to be unity for all the investigated systems. 


\begin{abstract}
Figure 14 Receiver efficiency as a function of absorber temperature for a blackbody absorber (solid black curve), a blackbody absorber with an optical cavity (this work, solid blue curve), an ideal spectrally selective absorber with a step function absorptivity from 1 to 0 at an optimized wavelength (dashed green curve) and a more realistic spectrally selective absorber with a step function absorptivity from 0.95 to 0.05 at $1.8 \mu \mathrm{m}$ wavelength (dotted red curve). These results assume AM1.5 Direct + Circumsolar illumination with a solar flux concentration ratio of 100 .
\end{abstract}

As expected, a blackbody absorber by itself is only effective at lower temperatures. The ideal spectrally selective absorber outperforms the blackbody absorber with the optical cavity at all temperatures, however the ideal characteristics used for the ideal absorber would be very difficult to achieve in practice. Comparing the cavity to the more realistic $95 / 5$ spectrally selective absorber, one can see that the two systems have very similar performance in the temperature range of $700{ }^{\circ} \mathrm{C}-1000^{\circ} \mathrm{C}$. At temperatures below $700^{\circ} \mathrm{C}$ the spectrally selective absorber performs worse due to absorptance being below unity. At temperatures greater than $1000{ }^{\circ} \mathrm{C}$ the proposed receiver design outperforms the $95 / 5$ spectrally selective absorber due to the overlap between the solar and IR spectra increasing the emittance of the spectrally selective absorber at high temperature. It is worth noting that this is a favorable treatment of spectrally selective absorbers, as in practice they do not have ideal step function spectral absorptances and are typically not stable at temperatures above $600{ }^{\circ} \mathrm{C}$ (Selvakumar and Barshilia, 2012). Thus, it is readily apparent that the optical cavity provides a significant advantage over a simple blackbody absorber, and can outperform spectrally selective surfaces at low and very high temperatures.

\title{
5 Conclusion
}

A specularly reflecting hemispherical cavity was proposed as a means to achieve directional selectivity for a solar receiver. Ray tracing simulations showed that the use of the cavity could reduce radiative losses from a blackbody surface by over $75 \%$, if the absorber could be precisely aligned within a large 
cavity. Results from proof of concept experiments demonstrated significant reductions in radiative losses that were reasonably matched with simulation results. These reductions in radiative losses indicate that the proposed optical cavity could potentially improve the efficiency of solar thermal systems without increased heat fluxes and their associated challenges. Potential future directions include the integration of this cavity concept into a solar thermal prototype system to characterize efficiency improvements and the investigation of a cylindrical cavity for use with line focus solar thermal systems.

\section{Acknowledgements}

The authors would like to thank Jonathan Rea for assistance in fabricating the experimental setup, and Professor Z.F. Ren's group at the University of Houston for helpful discussions. This work is supported by "Concentrated Solar Thermoelectric Power", a DOE SunShot CSP grant, under award number DEEE0005806.

\section{References}

ASTM G, 2003. Standard Tables for Reference Solar Spectral Irradiances: Direct Normal and Hemispherical on $37^{\circ}$ Tilted Surface. 2ASTM. doi:10.1520/G0173-03R08

Badescu, V., 2005. Spectrally and angularly selective photothermal and photovoltaic converters under one-sun illumination. J. Phys. D. Appl. Phys. 38, 2166-2172. doi:10.1088/0022-3727/38/13/014

Baranowski, L.L., Snyder, G.J., Toberer, E.S., 2012. Concentrated solar thermoelectric generators. Energy Environ. Sci. 5, 9055. doi:10.1039/c2ee22248e

Bermel, P., Lee, J., Joannopoulos, J.D., Celanovic, I., Soljačić, M., 2012. Selective Solar Absorbers, in: Annual Review of Heat Transfer Vol. 15. Begell House, pp. 231-254.

Blanco, M.J., Martín, J.G., Alarcón-Padilla, D.C., 2004. Theoretical efficiencies of angular-selective nonconcentrating solar thermal systems. Sol. Energy 76, 683-691. doi:10.1016/j.solener.2004.01.005

Boriskina, S. V., Chen, G., 2014. Exceeding the solar cell Shockley-Queisser limit via thermal upconversion of low-energy photons. Opt. Commun. 314, 71-78. doi:10.1016/j.optcom.2013.10.042

Brandt, R., Bird, C., Neuer, G., 2008. Emissivity reference paints for high temperature applications. Measurement 41, 731-736. doi:10.1016/j.measurement.2007.10.007

Braun, A., Katz, E.A., Feuermann, D., Kayes, B.M., Gordon, J.M., 2013. Photovoltaic performance enhancement by external recycling of photon emission. Energy Environ. Sci. 6, 1499. doi:10.1039/c3ee40377g

Cao, F., McEnaney, K., Chen, G., Ren, Z., 2014. A review of cermet-based spectrally selective solar absorbers. Energy Environ. Sci. 7, 1615. doi:10.1039/c3ee43825b 
Crabtree, G.W., Lewis, N.S., 2007. Solar energy conversion. Phys. Today.

Fernández-García, A., Zarza, E., Valenzuela, L., Pérez, M., 2010. Parabolic-trough solar collectors and their applications. Renew. Sustain. Energy Rev. 14, 1695-1721. doi:10.1016/j.rser.2010.03.012

Florescu, M., Lee, H., Puscasu, I., Pralle, M., Florescu, L., Z. Ting, D., Dowling, J.P., 2007. Improving solar cell efficiency using photonic band-gap materials. Sol. Energy Mater. Sol. Cells 91, 1599-1610. doi:10.1016/j.solmat.2007.05.001

Gordon, J.M., Feuermann, D., Huleihil, M., Katz, E.A., 2004. New optical systems for the solar generation of nanomaterials, in: Winston, R. (Ed.), Optical Science and Technology, SPIE's 48th Annual Meeting. International Society for Optics and Photonics, pp. 99-108. doi:10.1117/12.498858

Hamam, R.E., Celanovic, I., Soljačić, M., 2011. Angular photonic band gap. Phys. Rev. A 83, 035806. doi:10.1103/PhysRevA.83.035806

Harris, J., Lenz, T., 1985. Thermal performance of solar concentrator/cavity receiver systems. Sol. energy 34, 135-142.

Hiller, J., Mendelsohn, J.D., Rubner, M.F., 2002. Reversibly erasable nanoporous anti-reflection coatings from polyelectrolyte multilayers. Nat. Mater. 1, 59-63. doi:10.1038/nmat719

Hollands, K.G.T., 1963. Directional selectivity, emittance, and absorptance properties of vee corrugated specular surfaces. Sol. Energy 7, 108-116. doi:10.1016/0038-092X(63)90036-7

Howell, J.R., 1998. The Monte Carlo Method in Radiative Heat Transfer. J. Heat Transfer 120, 547. doi:10.1115/1.2824310

Kraemer, D., Poudel, B., Feng, H.-P., Caylor, J.C., Yu, B., Yan, X., Ma, Y., Wang, X., Wang, D., Muto, A., McEnaney, K., Chiesa, M., Ren, Z., Chen, G., 2011. High-performance flat-panel solar thermoelectric generators with high thermal concentration. Nat. Mater. 10, 532-8. doi:10.1038/nmat3013

Lenert, A., Bierman, D.M., Nam, Y., Chan, W.R., Celanović, I., Soljačić, M., Wang, E.N., 2014. A nanophotonic solar thermophotovoltaic device. Nat. Nanotechnol. 9, 126-30. doi:10.1038/nnano.2013.286

Luque, A., Araújo, G., 1990. Physical limitations to photovoltaic energy conversion. Adam Hilger, Bristol.

Mills, D., 2004. Advances in solar thermal electricity technology. Sol. Energy 76, 19-31. doi:10.1016/S0038-092X(03)00102-6

Modest, M.F., 2013. Radiative Heat Transfer, Third. ed. Academic Press.

Palmer, J., 1995. The measurement of transmission, absorption, emission, and reflection. Handb. Opt. 125. 
Perlmutter, M., Howell, J.R., 1963. A Strongly Directional Emitting and Absorbing Surface. J. Heat Transfer 85, 282. doi:10.1115/1.3686103

Prakash, M., Kedare, S.B., Nayak, J.K., 2009. Investigations on heat losses from a solar cavity receiver. Sol. Energy 83, 157-170. doi:10.1016/j.solener.2008.07.011

Selvakumar, N., Barshilia, H.C., 2012. Review of physical vapor deposited (PVD) spectrally selective coatings for mid- and high-temperature solar thermal applications. Sol. Energy Mater. Sol. Cells 98, 1-23. doi:10.1016/j.solmat.2011.10.028

Shen, Y., Ye, D., Celanovic, I., Johnson, S.G., Joannopoulos, J.D., Solja i , M., 2014. Optical Broadband Angular Selectivity. Science (80-. ). 343, 1499-1501. doi:10.1126/science.1249799

Simon, F.F., 1976. Flat-plate solar-collector performance evaluation with a solar simulator as a basis for collector selection and performance prediction. Sol. Energy 18, 451-466. doi:10.1016/0038092X(76)90012-8

Smil, V., 2005. Energy at the Crossroads: Global Perspectives and Uncertainties. MIT Press.

Steinfeld, a., Schubnell, M., 1993. Optimum aperture size and operating temperature of a solar cavityreceiver. Sol. Energy 50, 19-25. doi:10.1016/0038-092X(93)90004-8

Weinstein, L.A., 2013. Improvements to solar thermoelectric generators through device design. Massachusetts Institute of Technology.

Winston, R., Minano, J.C., Benitez, P.G., Shatz, N., Bortz, J.C., 2005. Nonimaging Optics. Elsevier Academic Press, Burlington, MA. 